\title{
The Hawthorne effect in
}

\section{measurements of hand hygiene compliance: a definite problem, but also an opportunity}

\author{
Sarah Haessler
}

Correspondence to

Dr Sarah Haessler, Tufts University School of Medicine, Baystate Medical Center, Baystate Infectious Diseases Division, 759 Chestnut Street, Springfield, MA 01199, USA; Sarah.Haessler@baystatehealth. org

Accepted 21 August 2014 Published Online First

11 September 2014

\section{SLinked}

http://dx.doi.org/10.1136/ bmjqs-2014-003080

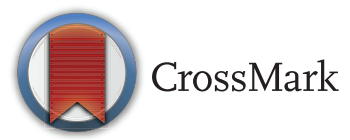

To cite: Haessler S. BMJ Qual Saf 2014;23:965-967.
The Hawthorne effect was first described in the 1950s, based on an analysis of experiments conducted three decades earlier at the Hawthorne works of the Western Electric Company in Illinois. ${ }^{1}$ These investigations of ways to improve productivity show that regardless of the intervention-changes in factory illumination, changes in the structure of breaks or even changes back to the original working conditions-worker productivity increased. While many have debated the meaning of these results, ${ }^{2}{ }^{3}$ a common, basic interpretation has been that the attention received by participants in an experiment can itself alter the outcomes of the study.

The concept that subjects behave differently in experimental settings has had an enduring impact in social sciences research. ${ }^{3}$ In medical research, the Hawthorne effect is seen as a type of bias, as patients seem to fare better by simply participating in a clinical trial, presumably due to the increased attention paid to participants, and the benefits of being watched. ${ }^{4}$ The effect has been most convincingly demonstrated in a study of intense versus minimal follow-up among patients with dementia receiving Gingko supplements, showing that the act of frequent follow-up visits rather than the administration of a medication led to improved patient outcomes. ${ }^{5}$ Unlike the placebo effect, ${ }^{6}$ where patients in a placebo arm experience a real change in symptoms while taking an inert substance, the Hawthorne effect represents a social phenomenon driven by a desire to please and meet the expectations of the researcher. $^{7}$

In healthcare epidemiology, direct observation has long been the gold standard for monitoring hand hygiene compliance rates. Yet, many are worried that the Hawthorne effect inflates rates of hand hygiene compliance generated by direct observation. Healthcare workers will be more likely to perform hand hygiene when they know that a monitor is observing them. ${ }^{8}$ Data collected by unit-based hand hygiene auditors have especially been suspected because the auditor is easily recognisable and their mere presence on the unit could trigger a temporary increase in hand hygiene activity, which artificially inflates the compliance rate.

Although no study has convincingly demonstrated the Hawthorne effect in measuring hand hygiene compliance, the plausibility of such an effect has stimulated various creative methods to avoid it and obtain more accurate measures. For instance, 'secret shoppers' not suspected of auditing hand hygiene, such as medical students, research assistants and hospital administrators, have been used at some hospitals. ${ }^{9-11}$ High tech methods of monitoring compliance while avoiding biased measurement have included radio frequency identification systems, wireless radio systems, video-monitored observation systems and electronic counters mounted on hand hygiene dispensers. ${ }^{12}$ However, these systems are expensive and carry the risk of eroding employee trust by generating the sense of 'Big Brother watching'. Thus, the question of whether or not the Hawthorne effect inflates rates of hand hygiene compliance obtained by standard observation remains an important one for most hospitals.

Srigley et $a l^{12}$ used a real-time location system (RTLS) to track the usage of hand hygiene products and the location of 
hand hygiene auditors over an 8-month period in two inpatient units at an academic medical centre. The RTLS used small ultrasound-emitting tags worn by staff who volunteered to take part in the study. The system continuously monitored the location of staff using a network of wireless receivers in patient rooms and hallways, and above all alcohol-based hand rub and soap dispensers. A geographical information systems engine used the ultrasound emissions from each tag to compute movement, location and proximity of tags to each other.

Healthcare workers and auditors knew about a larger study of the impact of a type of behaviour change approach (positive deviance) on healthcare associated infections. However, they remained blinded to a sub-hypothesis of the study aiming to detect the Hawthorne effect in relation to the use of hand hygiene product on exposure to an auditor. Specifically, this sub-study sought to measure the impact of an auditor within eyesight on the frequency of hand hygiene events. Using the layout of the study units and the placement of sensors, the authors identified the line of sight relative to an auditor at any given time. They then compared hand hygiene events for staff within eyesight of an auditor with event rates when the auditor was not present at all or present but not within eyesight.

The study showed an impressive threefold increase in the hand hygiene event rate when the auditor was in the line of sight of a dispenser, ${ }^{12}$ as compared with the same dispenser 1 week earlier (with no auditor present) and with dispensers on the same unit at the same time that were not within the line of sight of the auditor. Not surprisingly, the increased event rate did not extend to the dispensers located inside patient rooms, which is where most of the 'five moments' for hand hygiene proposed by the WHO occur. ${ }^{13}$ Since the Hawthorne effect appears to occur at the most public and visible point of care (entry or exit to a room), it follows that the other moments, which generally occur in the privacy of a patient's room, would have even lower compliance.

While the results of this study confirm the existence of an observer effect in hand hygiene, it is not a straightforward exercise to use the results of this study to estimate true rates of hand hygiene when no auditor is on the unit. Only a minority of staff volunteered for the study. The number of staff wearing the RTLS tags was sufficient to generate statistical significance for the threefold increase in the likelihood of hand hygiene compliance within the eyesight of an auditor. But, the number of staff wearing RTLS tags at any given time was too small to generate a reliable estimate of hand hygiene compliance in the absence of auditors. In addition, some individual healthcare workers have higher baseline rates of compliance than others. However, regardless of whether individual healthcare workers were high or low performers, the authors were able to demonstrate over time an impressive overall impact on those wearing a sensor when auditors stepped onto the unit. While the threefold increase in hand hygiene adherence represents a robust result, it does not follow that hospitals must divide their observed hand hygiene compliance by a factor of 3 in order to estimate the true rate of hand hygiene compliance. In other words, while the results of Srigley et al convincingly demonstrate that the presence of an auditor increases the likelihood of hand hygiene compliance by a factor of 3 , it is not the case that a hospital that has an apparently high hand hygiene compliance rate of, say, 90\%, in fact has a dismal rate of $30 \%$.

How could this be? The compliance rate observed by auditors includes observations of some staff who perform hand hygiene most of the time (70\%-90\%) and some who have much lower adherence $(20 \%-$ $40 \%)$. What Srigley et $a l^{12}$ show is that both these types of people (call them adherers and non-adherers) are more likely to engage in hand hygiene when an auditor is within eyesight. The magnitude of the bias introduced by the Hawthorne effect depends on the proportion of non-adherers.

How to apply the results of this study to one's own hospital depends on the intensity and sophistication of the hand hygiene programme within an institution. If an organisation with a hand hygiene compliance rate of $40 \%$ engages in a simple audit and feedback programme - posting monthly hand hygiene rates on units-along with a memo or two from hospital leaders is shown to have a rate of $90 \% 6$ months later; this dramatic increase likely represents a substantially biased estimate due to the Hawthorne effect. Most of the staff in this hospital are non-adherers. Their hand hygiene adherence rate increases substantially when known observers are on the unit. The true rate of adherence is probably the rate when no observer is present, since the impact of an observer is short-lived and only serves as a temporary reminder to healthcare workers that they should be cleaning their hands. By contrast, in a hospital with a more intensive programme, including not just audit and feedback but a major education campaign, increased signage, maximal product placement, computer screensavers, unit-based champions and other methods aimed at changing culture, a steady rise in adherence rates over 1-2 years has likely achieved sustained improvement in the true unobserved hand hygiene rate. ${ }^{14}$

The results of this study ${ }^{12}$ help us recognise that sometimes gains in hand hygiene rates are 'too good to be true.' Humans are social beings who aim to please and wish to appear better than they are. Simply reporting observed hand hygiene rates back to staff is not likely to cause major improvements. If major improvements are observed, they are probably spurious and reflect a Hawthorne effect. In such cases, the hospital needs to undertake more intensive interventions that address the causes of poor compliance, and 
possibly supplementing routine observation with other measurement methods.

Instead of lamenting that the Hawthorne effect in hand hygiene documented by Srigley et $a l^{12}$ requires us to downwardly readjust our expectations of compliance, their results should lead us to consider ways in which this phenomenon can be harnessed. Actively appealing to the human desires for positive feedback and conformity to workplace norms provides the opportunity to harness the Hawthorne effect. Implementing intensive strategies that play on the awareness of being observed, such as regularly announcing audits, rotating auditors, embedding auditors into care teams or high tech tracking devices, are tools that are more likely to drive sustained change. By understanding how the Hawthorne effect impacts hand hygiene behaviour, we can appropriately interpret our hand hygiene rates and use the power of being watched and the desire to please as tools in our efforts to improve patient safety and organisational culture.

\section{Competing interests None.}

Provenance and peer review Not commissioned; internally peer reviewed.

\section{REFERENCES}

1 Adair JG. The Hawthorne effect: a reconsideration of the methodological artifact. J Appl Psychol 1984;69:334-45.

2 Jones SRG. Was there a Hawthorne effect?. Am J Sociol 1992;98:451-68.

3 Levitt SD, List JA. Was there really a Hawthorne effect at the Hawthorne plant? An analysis of the original illumination experiments. Am Econ J Appl Econ 2011;3:224-38.
4 Braunholtz DA, Edwards SJ, Lilford RJ. Are randomized clinical trials good for us (in the short term)? Evidence for a "trial effect". J Clin Epidemiol 2001;54:217-24.

5 McCarney R, Warner J, Iliffe S, et al. The Hawthorne effect: a randomised, controlled trial. BMC Med Res Methodol 2007;7:30.

6 de Craen AJ, Kaptchuk TJ, Tijssen JG, et al. Placebos and placebo effects in medicine: historical overview. J R Soc Med 1999;92:511-15.

7 Berthelot JM, Le Goff B, Maugars Y. The Hawthorne effect: stronger than the placebo effect? Joint Bone Spine 2011;78:335-6.

8 Haas JP, Larson EL. Measurement of compliance with hand hygiene. J Hosp Infect 2007;66:6-14.

9 Dhar S, Tansek R, Toftey EA, et al. Observer bias in hand hygiene compliance reporting. Infect Control Hosp Epidemiol 2010;31:869-70.

10 Ellingson K, Haas JP, Aiello AE, et al. Strategies to prevent healthcare-associated infections through hand hygiene. Infect Control Hosp Epidemiol 2014;35:937-60.

11 Pan SC, Tien KL, Hung IC, et al. Compliance of health care workers with hand hygiene practices: independent advantages of overt and covert observers. PLOS ONE 2013; 8:e53746.

12 Srigley JA, Furness CD, Baker GR, et al. Quantification of the Hawthorne effect in hand hygiene compliance monitoring using an electronic monitoring system: a retrospective cohort study. BMJ Qual Saf 2014;23:974-80.

13 WHO guidelines on hand hygiene in health care. 2009. http:// www.who.int/gpsc/5may/tools/9789241597906/en/ (accessed 15 Aug 2014).

14 Kirkland KB, Homa KA, Lasky RA, et al. Impact of a hospital-wide hand hygiene initiative on healthcare-associated infections: results of an interrupted time series. BMJ Qual Saf 2012;21:1019-26. 